\title{
Cáncer de la unión gastroesofágica. Evaluación de los resultados quirúrgicos, sobrevida alejada y factores pronósticos en enfermos con terapia resectiva
}

\author{
JEAN M. BUTTE ${ }^{1}$, FEDERICO BECKER ${ }^{2 a}$, ÁLVARO VISSCHER ${ }^{2 a}$, \\ ENRIQUE WAUGH' ${ }^{1}$, MANUEL MENESES ${ }^{1}$, ISMAEL COURT ${ }^{1}$, \\ HUGO PARADA ${ }^{1}$, HERNÁN DE LA FUENTE ${ }^{1}$
}

\section{Adenocarcinoma of the esophagogastric junction. Retrospective analysis of 39 patients}

\begin{abstract}
Background: The long term survival of adenocarcinoma of the esophagogastric junction is poor and depends on the possibility of performing a complete surgical excision and the absence of lymph node involvement. Aim: To report surgical results and survival of patients with adenocarcinoma of the esophagogastric junction. Material and Methods: Retrospective review of medical records of patients with adenocarcinoma of the esophagogastric junction, subjected to a curative surgical procedure between 2000 and 2008. Deaths that occurred within 60 days of the operation were considered operative mortality. Tumor stage was determined using TNM and Siewert pathological classifications. Results: Thirty nine patients aged 40 to 80 years (27 men), were operated. According to Siewert classification, seven patients had type I, six type II and 26 type III tumors. Twenty two patients were subjected to a total gastrectomy with partial excision of distal esophagus and mediastinal reconstruction, 10 patients were subjected to a transhiatal esophagectomy and seven to a total esophagogastrectomy. According to postoperative staging, five patients were in stage I, 12 in stage II, nine in stage III and 13 in stage IV. Median, three and five years survival figures were 21.4 months, 33 and 25\%, respectively. Lymph node and perineural involvement was associated with a lower survival. Well differentiated and stage I tumors had a better survival. Multivariate analysis showed that the presence of a type III tumor, N3 lymph node involvement and vascular permeation were independent predictors of a lower survival. Conclusions: Among patients with adenocarcinoma of the esophagogastric junction, type III tumors, lymph node involvement and vascular permeations are associated with a lower survival.
\end{abstract}

(Rev Med Chile 2010; 138: 53-60).

Key words: Adenocarcinoma; Esophageal neoplasms; General surgery.

$\mathrm{L}$ os adenocarcinomas de la unión gastroesofágica incluyen a los tumores originados en el esófago distal con invasión de la unión gastroesofágica, a los tumores cardiales verdaderos y a los tumores originados en el estómago proximal con invasión de la unión gastroesofágica ${ }^{1}$. Debido al diferente origen y patrón de diseminación, no existe un sistema de etapificación TNM específico. A pesar de esto, la clasificación topográfica propuesta por Siewert ${ }^{2}$ es la más utilizada. En esta, los tumores se dividen en tipo I, II y III, si el origen primario es en el esófago distal, la unión gastroesofágica propiamente tal o el estómago proximal, respectivamente.
'Instituto Oncológico Fundación Arturo López Pérez (FALP) Santiago, Chile. ${ }^{2}$ Universidad de los Andes. anterno de Medicina.

Recibido el 21 de julio de 2009, aceptado el 14 de diciembre de 2009.

Correspondecia a: Dr. Hernán De La Fuente $\mathrm{H}$. Instituto Oncológico Fundación Arturo López Pérez Rancagua 878 Santiago, Chile Teléfono: 56-2-4457247 Fax: 56-2-4218597 E-mail: delafueh@yahoo.com 
En los últimos años se ha producido un aumento en la incidencia del cáncer de la unión gastroesofágica $(\mathrm{CUGE})^{3}$. La mayor prevalencia del esófago de Barrett, asociado al aumento del reflujo gastroesofágico (RGE) y la obesidad, han contribuido a este incremento ${ }^{4}$.

El tratamiento del CUGE es complejo. Hay consenso en que en ausencia de enfermedad metastásica, la resección quirúrgica es el tratamiento de elección. Sin embargo, la sobrevida alejada con cirugía exclusiva, rara vez sobrepasa el $20 \%$ a 5 años. Por ello, la asociación de terapias neoadyuvantes y adyuvantes ha sido explorada con interés. Con los tratamientos neoadyuvantes no sólo ha aumentado la resecabilidad sino que también la sobrevida global y libre de enfermedad. Con radioquimioterapia adyuvante se ha descrito un mejor control local y un aumento en la sobrevida global y libre de enfermedad ${ }^{5,6}$. Pese a esto, las características metodológicas y los diferentes sesgos observados en estos estudios hacen que aún no se puedan obtener evidencias definitivas y concluyentes que sustenten su uso como una terapia estándar?

Tampoco existe consenso sobre la técnica resectiva ideal ${ }^{3,8}$. Las alternativas quirúrgicas más utilizadas han sido: 1) esofagectomía transhiatal más resección gástrica proximal y ascenso gástrico con anastomosis intramediastínica o cervical, 2) gastrectomía total con resección esofágica distal y anastomosis esofagoyeyunal intramediastínica y 3 ) esofagectomía distal más gastrectomía proximal y anastomosis esofagogástrica intramediastínica. Con menos frecuencia se utiliza la esofagogastrectomía total con interposición de colon y anastomosis esofagocolónica cervical ${ }^{9}$.

Los objetivos de este estudio son evaluar los resultados del tratamiento quirúrgico, la sobrevida alejada e identificar factores pronósticos asociados a la sobrevida en un grupo de enfermos con un adenocarcinoma de la unión gastroesofágica tratados con cirugía resectiva.

\section{Material y Métodos}

Pacientes: Se realizó un análisis retrospectivo a partir de los registros clínicos y anatomopatológicos de los pacientes sometidos a la resección quirúrgica de un adenocarcinoma de la unión gastroesofágica entre el 1 de enero de 2000 y el 30 de junio de 2008 en el Instituto Oncológico Fundación Arturo López Pérez. Los datos fueron recopilados retrospectivamente hasta el 31 de mayo de 2007 y prospectivamente desde el 1 de junio de 2007. En cada paciente se analizaron las características clínicas, el tratamiento administrado, el estudio anatomopatológico y la sobrevida alejada.

Se consideró mortalidad operatoria a la ocurrida hasta 60 días después de la operación y asociada en forma directa con el procedimiento quirúrgico. Todos los enfermos fueron seguidos hasta la fecha de su muerte o hasta el cierre de este estudio. El estadio tumoral fue determinado mediante la clasificación TNM del año 2002 para cáncer gástrico y esofágico ${ }^{10}$.

Evaluación preoperatoria: Todos los enfermos fueron evaluados y tratados con el mismo protocolo y por el mismo equipo quirúrgico. En cada paciente se realizó una endoscopía digestiva alta que confirmó la ubicación tumoral y permitió obtener material para estudio anatomopatológico. El estudio de diseminación se realizó con una tomografía computada de tórax, abdomen y pelvis o una tomografía de emisión de positrones con fluordeoxiglucosa ${ }^{18}\left({ }^{18} \mathrm{FD}\right.$ PET-CT). No se realizó estudio radiológico contrastado de rutina. Todos los pacientes recibieron una evaluación preoperatoria de su capacidad cardiovascular, renal, respiratoria y nutricional. Se agregó una colonoscopía completa, para descartar un segundo primario y por la eventual necesidad de utilizar el colon para reconstituir el tránsito digestivo.

Tratamiento quirúrgico administrado: El día previo a la operación se realizó una preparación intestinal con polietilenglicol. Durante la operación, los enfermos fueron posicionados para una eventual esofagectomía y gastrectomía total. Luego de descartar la presencia de enfermedad metastásica en el abdomen, se evaluó la ubicación tumoral, y se decidió el tipo de tratamiento quirúrgico, así como la forma de reconstrucción del tránsito intestinal. La elección de la técnica a utilizar se basó en hechos clínicos como la ubicación topográfica de la lesión, la cuantía del compromiso esofágico, la cuantía del compromiso ganglionar y la apreciación del riesgo perioperatorio que podía implicar la complejidad de la reconstrucción a utilizar. De acuerdo a esto se practicó: (A) gastrectomía total, esofagectomía distal y reconstrucción con esófago-yeyuno anastomosis intramediastínica, (B) esofagectomía transhiatal y reconstrucción con 
ascenso gástrico y anastomosis esófago-gástrica cervical o (C) esofagectomía total, gastrectomía total y reconstrucción con ascenso de colon (por vía retroesternal o mediastino posterior) y anastomosis esófago-colónica cervical. En los pacientes sometidos a una gastrectomía total (procedimiento A) se realizó una linfadenectomía abdominal D2 $\mathrm{y}$ del mediastino inferior, en aquellos sometidos a una esofagectomía transhiatal (procedimiento B) se resecaron los grupos abdominales 1, 2, 3 y 7 y los del mediastino infracarinal, junto al esófago. En los enfermos sometidos a una esofagectomía total y gastrectomía total (procedimiento C), se realizo una linfadenectomía abdominal D2 y del mediastino infracarinal junto a la esofagectomía. La anastomosis esófago-yeyunal se realizó término-lateral, con una engrapadora circular $\mathrm{N}^{\circ} 21$ ó 25, de acuerdo a las características del paciente y decisión del cirujano. La anastomosis se cubrió con un segundo plano seroso-muscular con monocryl ${ }^{\circledR} 4-0$ continuo. La anastomosis esófagogástrica cervical se realizó en forma manual con monocryl ${ }^{\circledR} 4-0$ en forma continua. La anastomosis esófago-colónica cervical se realizó con monocryl ${ }^{\circledR}$ 4-0 en forma continua. Todas las otras anastomosis abdominales se realizaron en forma manual con monocryl ${ }^{\circledR}$ 4-0 en forma continua. En todos los enfermos se realizó una colecistectomía y una apendicectomía cuando estos órganos estuvieron presentes. No se realizó esplenectomía de rutina.

Estudio anatomopatológico: La pieza operatoria fue evaluada durante la operación por el mismo patólogo, que confirmó la ausencia de compromiso tumoral en los márgenes quirúrgicos. Todas las piezas operatorias se clasificaron de acuerdo a lo descrito por Siewert ${ }^{2}$.

Tratamiento neoadyuvante/adyuvante: La administración de terapias multimodales (neoadyuvante o adyuvante) fue decidida de acuerdo al protocolo institucional previamente establecido. Los pacientes con lesiones localmente avanzadas y potencialmente no resecables en forma completa, fueron sometidos a quimioterapia neoadyuvante y evaluación post tratamiento. Fueron explorados sólo los pacientes con respuesta objetiva o enfermedad estable sin evidencias de compromiso metastásico. Algunos pacientes resecados recibieron quimioterapia o radioquimioterapia adyuvante. El esquema de quimioterapia perioperatoria incluye epirubicina, cisplatino y 5 -fluoruracilo. Después de tres ciclos los enfermos fueron ree- valuados y aquellos que presentaron reducción o estabilización tumoral fueron seleccionados para tratamiento quirúrgico. Posterior a la resección quirúrgica son presentados al comité institucional. El tratamiento adyuvante se define de acuerdo a los hallazgos intraoperatorios y la información de la biopsia postoperatoria. El esquema de radio-quimioterapia adyuvante consiste en un ciclo de 5-fluoruracilo $\left(425 \mathrm{mg} / \mathrm{m}^{2}\right.$ por día en infusión continua) más leucovorina $\left(20 \mathrm{mg} / \mathrm{m}^{2}\right.$ endovenoso) por 5 días previo a la radioterapia. Posteriormente se administra en forma concomitante a la radioterapia, los primeros 4 y últimos 3 días. Durante este periodo se disminuye la dosis de 5 -fluoruracilo a $400 \mathrm{mg} / \mathrm{m}^{2}$ por día. Posterior a la radioterapia, se administran 2 ciclos más de quimioterapia.

Estadística: El análisis estadístico incluyó test de Student, Mann-Whitney, $\chi^{2}$ o prueba de Fisher. Las curvas de sobrevida se obtuvieron con el método de Kaplan-Meier y las diferencias fueron comparadas con el test de log-Rank. Se realizó un análisis multivariado de riesgos proporcionales con el método de Cox. Se consideró significativo un $\mathrm{p}<0,05$.

\section{Resultados}

Durante el período analizado se operaron 40 pacientes. En 39 de ellos se logró una resección completa del tumor y son los pacientes que se evalúan en este estudio. Un paciente no fue resecado por carcinomatosis peritoneal. De esta forma, el grupo de estudio está formado por 27 hombres y 12 mujeres con una edad promedio de 63 años (i: 40-80 años).

Se realizó una gastrectomía total y esofagectomía distal a 22 pacientes, una esofagectomía transhiatal a 10 y una esofagectomía total y gastrectomía total a 7 pacientes (Figura 1).

Se presentaron una o más complicaciones en 17 pacientes (41\%) (Tabla 1). Dos de ellos $(5,1 \%)$ fueron reoperados; uno por sangrado postoperatorio y otro por una hernia transhiatal aguda con necrosis intestinal. No hubo mortalidad postoperatoria.

El estudio de anatomía patológica confirmó 7 casos de tumores tipo I de Siewert, 6 de tipo II y 26 de tipo III. En los pacientes con tumores tipo I, 2 enfermos presentaron compromiso de la mucosa, 
Tabla 1. Morbilidad postoperatoria

\begin{tabular}{|lcc|}
\hline Morbilidad & $\mathbf{N}^{\circ}$ & $\%$ \\
\hline Arritmia supraventricular & 3 & 7,7 \\
\hline Tromboembolismo pulmonar & 2 & 5,1 \\
\hline Atelectasias & 2 & 5,1 \\
\hline Síndrome febril sin foco & 2 & 5,1 \\
\hline Derrame pleural & 2 & 5,1 \\
\hline Disfonía transitoria & 2 & 5,1 \\
\hline Retención gástrica & 2 & 5,1 \\
\hline Neumonía & 2 & 5,1 \\
\hline Sangrado arterial* & 1 & 2,6 \\
\hline Necrosis intestinal por hernia transhiatal* & 1 & 2,6 \\
\hline Neumotórax & 1 & 2,6 \\
\hline Trombosis venosa profunda post alta & 1 & 2,6 \\
\hline Infección de la herida operatoria & 1 & 2,6 \\
\hline
\end{tabular}

*Pacientes reoperados.

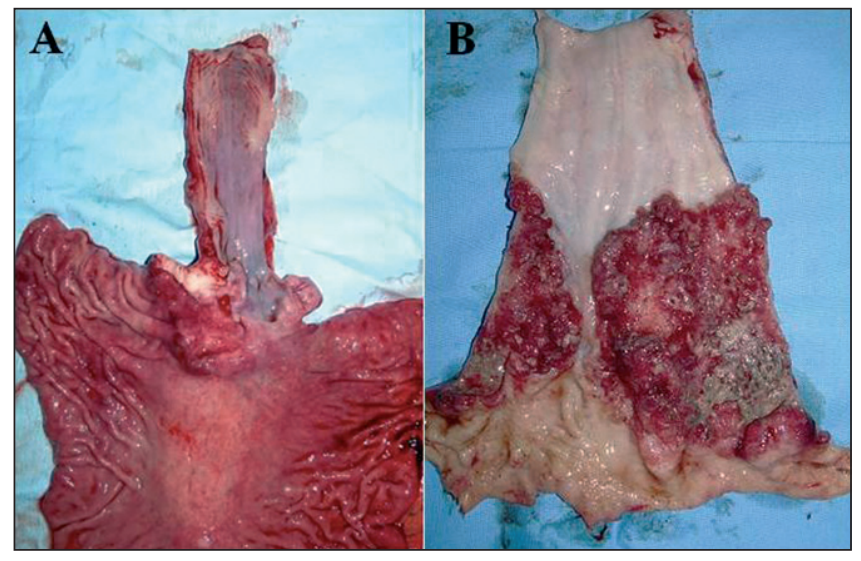

Figura 1. A: Gastrectomía total y esofagectomía total. B: Esofagectomía total.
2 de la muscular y 3 de la adventicia. En los pacientes con tumores tipo II, uno presentó compromiso de la mucosa, 2 de la muscular, uno de la subserosa, uno de la adventicia y uno de la serosa. En los pacientes con tumores tipo III, 2 pacientes presentaron compromiso muscular, 9 de la subserosa y 15 de la serosa. La mediana de ganglios resecados fue de 24 (rango 10-53) y la mediana de ganglios positivos fue de 7 (rango 0-32). La relación entre el tratamiento quirúrgico y el tipo de tumor se observa en la Tabla 2.

De acuerdo a la clasificación TNM, 5 pacientes fueron clasificados en estadio I, 12 en estadio II, 9 en estadio III y 13 en estadio IV (N3 en 12 y M1 en 1).

Un paciente con un tumor tipo III recibió quimioterapia pre y postoperatoria, cinco con un tumor tipo III y uno con un tumor tipo I recibieron radioquimioterapia postoperatoria y un paciente con un tumor tipo II y otro con un tumor tipo III recibieron quimioterapia postoperatoria. En total, 9 pacientes recibieron algún tipo de terapia multimodal.

La mediana de sobrevida y la sobrevida estimada a 3 y 5 años de los 39 enfermos fue de 21,4 meses, 33,3\% y $25,2 \%$, respectivamente (Figura 2). La sobrevida a 3 años de los pacientes con un tumor tipo I, tipo II y tipo III fue de $53,3 \%, 53,3 \%$ y $24,8 \%$, respectivamente $(\mathrm{p}=\mathrm{NS})$. La sobrevida a 3 años de los enfermos con compromiso ganglionar fue significativamente inferior a la de aquellos sin compromiso ganglionar $(23,1 \%$

Tabla 2. Tipo de resección según tipo de tumor

\begin{tabular}{|lcccc|}
\hline Tipo de tumor & GT+ED & ETH & EG & Total \\
\hline Tipo I & 0 & 7 & 0 & 7 \\
\hline Tipo II & 3 & 3 & 0 & 6 \\
\hline Tipo III & 19 & 0 & 7 & 26 \\
\hline
\end{tabular}

GT + ED: Gastrectomía total + esofagectomía distal. ETH: Esofagectomía transhiatal. EG: Esofagectomía total + gastrectomía total. 


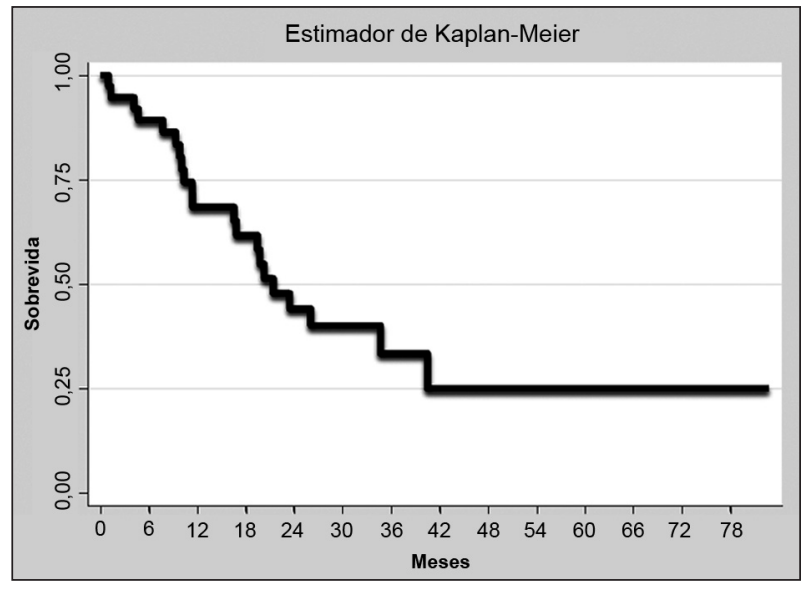

Figura 2. Sobrevida global de pacientes con un tumor de la unión gastroesofágica resecado $(n=39)$.

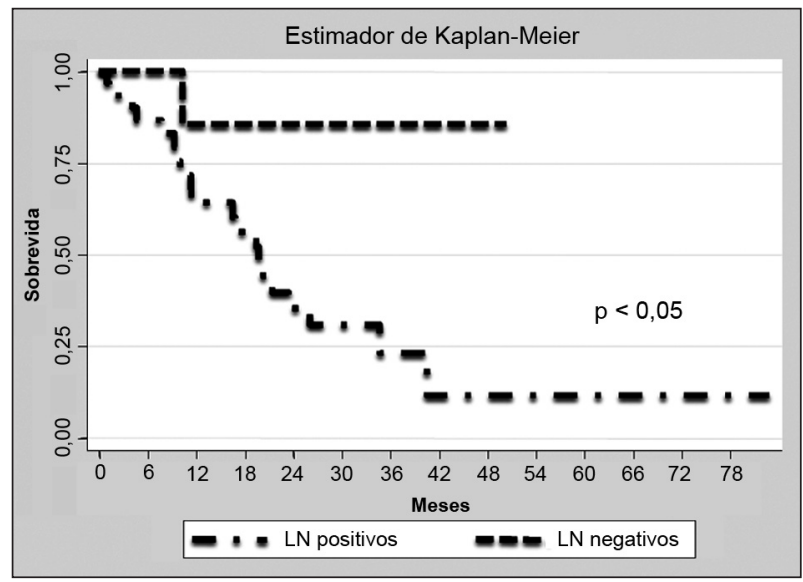

Figura 3. Sobrevida de pacientes con un tumor de la unión gastroesofágica resecado según compromiso ganglionar $(n=39)$.

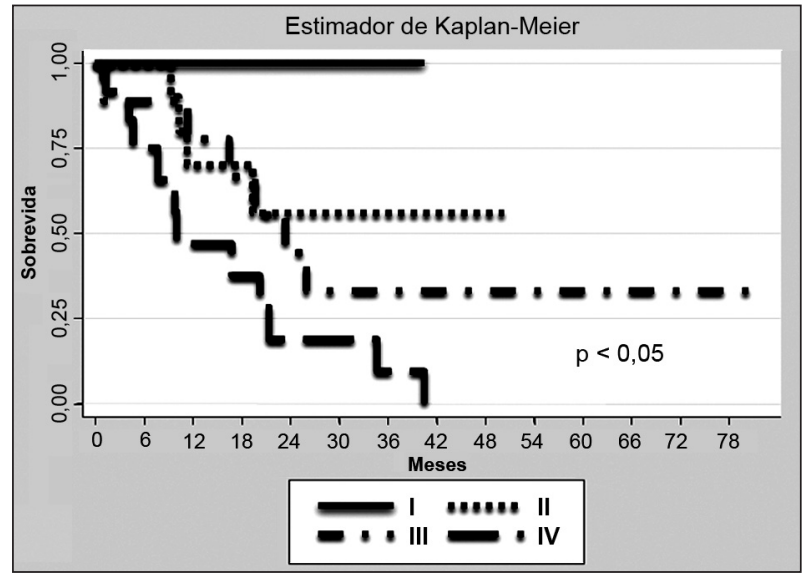

Figura 4. Sobrevida de pacientes con un tumor de la unión gastroesofágica resecado según etapa tumoral $(n=39)$. vs $85,7 \%)(\mathrm{p}<0,05)$ (Figura 3). La sobrevida a 3 años de los pacientes con compromiso ganglionar N3 fue significativamente inferior que la de aquellos con compromiso ganglionar $\mathrm{N} 1$ o $\mathrm{N} 2(8,5 \%$ vs $62,8 \%$ y $42,8 \%$, respectivamente $)(\mathrm{p}<0,05)$. La sobrevida a 3 años de los pacientes sin permeaciones vasculares fue significativamente mayor que la de aquellos enfermos con permeaciones vasculares $(47,6 \%$ vs $0 \%)(\mathrm{p}<0,05)$. La sobrevida de los enfermos en etapa I fue de $100 \%$, la de los en etapa II de $56 \%$, la de los en etapa III $33 \%$ y la de los etapa IV de $9 \%$ $(\mathrm{p}<0,05)$ (Figura 4).

El análisis univariado mostró que los pacientes con compromiso tumoral ganglionar, compromiso perineural y compromiso ganglionar de los grupos 1 ó 2 presentaron una peor sobrevida. Aquellos con tumores bien diferenciados y en etapa I, presentaron una mejor sobrevida.

El análisis bivariado mostró que los tumores tipo III, el compromiso ganglionar (versus sin compromiso ganglionar), el compromiso ganglionar $\mathrm{N} 3$ (versus compromiso ganglionar N1 ó N2) y las permeaciones vasculares se asociaron a peor sobrevida (Tabla 3).

El análisis multivariado mostró que el compromiso ganglionar N3 (versus compromiso ganglionar N1 ó N2) o permeaciones vasculares se asociaron a peor sobrevida (Tabla 4).

\section{Discusión}

El compromiso tumoral de la unión gastroesofágica se asocia a baja sobrevida global, compromiso tumoral loco-regional y la necesidad de procedimientos resectivos y reconstructivos complejos de alta morbilidad y mortalidad ${ }^{11}$. Independiente del sitio de origen (esofágico, cardias o estómago), la diseminación ganglionar es precoz, pudiendo comprometer los ganglios abdominales, mediastínicos e incluso cervicales ${ }^{12}$. A ello se agrega la frecuente infiltración tumoral esofágica, que puede extenderse a través de la submucosa más allá del límite oral palpable. En ocasiones, se han observado nidos 
Tabla 3. Análisis bivariado

\begin{tabular}{|lcc|}
\hline Característica & HR & IC 95\% \\
\hline Compromiso ganglionar N3 & 8,27 & $1,03-66,47$ \\
\hline Tumor tipo III & 4,89 & $1,05-22,63$ \\
\hline Permeaciones vasculares presentes & 2,78 & $1,05-7,32$ \\
\hline Compromiso ganglionar & 1,05 & $1-1,1$ \\
\hline
\end{tabular}

Tabla 4. Análisis multivariado

\begin{tabular}{|lcc|}
\hline Característica & HR & IC 95\% \\
\hline Compromiso ganglionar N3 & 9,84 & $1,21-80,02$ \\
\hline Permeaciones vasculares presentes & 3,22 & $1,16-8,9$ \\
\hline
\end{tabular}

tumorales submucosos, 5 a $10 \mathrm{~cm}$ proximales al límite oral de la lesión tumoral y sólo identificables en la revisión histológica de márgenes proximales sucesivos enviados a biopsia contemporánea (comunicación personal). Esta situación explicaría las recurrencias mediastínicas en pacientes sometidos a resecciones que no incluyeron el esofágo y los linfonodos mediastínicos y que en la biopsia contemporánea del margen oral no se detectó compromiso tumoral. La linfadenectomía debe incluir los territorios de drenaje linfático teórico que en el caso del CUGE implica la resección de al menos los linfonodos del mediastino inferior y del territorio abdominal perigástrico ${ }^{8}$. Ello implica realizar una esofagectomía total asociada a una gastrectomía total con linfadenectomía D2. Lamentablemente, la mayoría de los pacientes no tiene condiciones fisiológicas ni nutricionales para afrontar una extirpación y reconstrucción digestiva de tal envergadura, por lo que hay que ser selectivo en la aplicación de esta modalidad terapéutica ${ }^{3}$. Sin embargo, se observó que el riesgo perioperatorio de una esofagogastrectomía total no fue mayor que el de una gastrectomía total con anastomosis esófago-yeyunal intramediastínica. Por esto, podría ser razonable una resección más extensa si con ello se evita una reconstrucción de mayor morbilidad. De esta forma, el equipo quirúrgico debe estar familiarizado con todas las alternativas resectivas y con las distintas opciones y vías de reconstrucción del tránsito digestivo.

La morbilidad y mortalidad operatoria se asocia al volumen y experiencia del equipo qui- rúrgico $^{3,13}$. En esta serie, pese a que $41 \%$ de los pacientes tuvo alguna complicación perioperatoria y 2 pacientes fueron reoperados, no hubo filtraciones anastomóticas, necrosis de segmentos interpuestos ni mortalidad operatoria.

El estudio anatomopatológico intraoperatorio de los márgenes tumorales permitió sostener la decisión de realizar resecciones extendidas. El análisis debería ser realizado por un patólogo con experiencia en biopsia contemporánea e incluir varios cortes histológicos de los márgenes. El estudio anatomopatológico diferido, mostró que $66,6 \%$ de los pacientes presentaron un tumor originado en el estómago, tal como lo han señalado otros autores $^{8,14}$. Además, en 26,9\% de los enfermos con un tumor tipo III fue necesaria la resección completa del esófago por ascenso tumoral, compromiso nodal mediastínico, o ambos.

La mediana de sobrevida y la sobrevida global a 3 y 5 años de los enfermos tratados en esta serie fue de 21,4 meses, $33 \%$ y $25 \%$ respectivamente. Fue menor en los pacientes que presentaron un tumor tipo III o compromiso ganglionar, especialmente en aquellos con 15 o más ganglios comprometidos.

El análisis univariado, bivariado y multivariado mostraron resultados similares a los reportados en la literatura. Barbour y cols ${ }^{8}$, analizaron los resultados de 505 enfermos resecados por un CUGE y observaron que la mayoría de los tumores se originaron en el cardias o en el estómago, la mitad de los enfermos se encontraba en etapa III ó IV y la mediana de sobrevida fue de 27 meses. También se observó el ascenso tumoral a través del esófago, que llegó hasta $8 \mathrm{~cm}$ del borde del tumor primario. Fueron factores independientes de mal pronóstico el compromiso ganglionar N3, el compromiso parietal del tumor (peor en los tumores T3 ó T4), la mala diferenciación y un margen proximal menor a $3,8 \mathrm{~cm}$. Otro estudio, de Sihvo y cols ${ }^{15}$, en el que se analizaron 402 enfermos, mostró una sobrevida global a 5 años de $29 \%$. La cirugía radical R0 asociada a disección linfática en tres campos fue la que mostró los mejores resultados. Una revisión más reciente de Von Rahden y cols ${ }^{18}$, que evaluó más de 1500 casos consecutivos, confirmó que el factor pronóstico más importante asociado a sobrevida es la resección R0 con disección ganglionar.

La sobrevida a 5 años del CUGE es discreta. En los últimos años se han evaluado tratamientos neoadyuvantes y adyuvantes intentando mejorar 
Cáncer de la unión gastroesofágica - J. M. Butte et al

la sobrevida global. Los beneficios teóricos del tratamiento neoadyuvante serían aumentar la probabilidad de la resección quirúrgica al disminuir el tamaño tumoral, eliminar las micrometastasis, disminuir los síntomas producidos por el tumor y determinar la sensibilidad del tumor a la quimioterapia, lo que se consideraría un factor pronóstico de sobrevida ${ }^{6,17}$. El estudio MAGIC evaluó 503 enfermos en forma prospectiva, aleatorizada y multicéntrica. Se incluyeron pacientes con un adenocarcinoma originado en el estómago, la unión gastroesofágica o el esófago distal, al menos en etapa II, sin metástasis a distancia o con enfermedad localmente avanzada no resecable en la evaluación imagenológica inicial. Se distribuyó aleatoriamente a cirugía más quimioterapia perioperatoria y sólo cirugía. El principal objetivo evaluado fue la sobrevida global. Este estudio mostró que los enfermos que recibieron quimioterapia perioperatoria tuvieron una mayor sobrevida global y libre de enfermedad al compararlos con los pacientes tratados sólo con cirugía. Aunque este estudio ha recibido reparos metodológicos porque concentró estadios tumorales más precoces en el grupo de quimioterapia perioperatoria, porque sólo $40 \%$ de los pacientes completó el tratamiento perioperatorio y porque la mayoría de los pacientes no recibió cirugía con disección ganglionar D2, la sobrevida global obtenida frente a cirugía exclusiva obliga a evaluar este tratamiento. La radioquimioterapia adyuvante ha sido utilizada después de cirugía resectiva óptima y suboptima. Los resultados del estudio INT $0116^{5}$ que reportó mejor sobrevida global y libre de enfermedad en los pacientes sometidos a radioquimioterapia postoperatoria también han sido cuestionados en su metodología y aplicación clínica ${ }^{7}$. En este estudio hay una pequeña cohorte de pacientes portadores de CUGE cuyos resultados específicos no han sido reportados. Sin embargo, se tiende a extrapolar los beneficios obtenidos en sobrevida global y libre de enfermedad del estudio completo a este grupo de pacientes. Es muy probable que esta indicación de radioquimioterapia postoperatoria sea poco sustentable mientras no exista un estudio específico consistente y definitivo.

En nuestro medio, la posibilidad de la mayoría de los pacientes de acceder a terapias neoadyuvantes, perioperatorias y adyuvantes es muy baja. Factores como la ausencia de estas modalidades terapéuticas en las guías de tratamiento oficia- les ${ }^{18}$, el alto costo, la alta tasa de estadios tumorales avanzados con grave deterioro fisiológico y nutricional asociado dificultan su aplicación clínica. Sin embargo, creemos que la mejoría en los resultados del tratamiento del CUGE estará intimamente ligada a la interacción entre terapias multimodales y cirugía oncológica de calidad en centros focalizados de alto volumen.

\section{Referencias}

1. Stein H, Feith M, Siewert L. Cancer of the esophagogastric junction. Surg Oncol; 2000; 9: 35-41.

2. Siewert L, Feith M, Werner M, Stein H. Adenocarcinoma of the esophagogastric junction. Results of surgical therapy based on Anatomical/Topographic classification in 1002 consecutive patients. Ann Surg 2000; 232: 353-61.

3. Demeester S. Adenocarcinoma of the esophagus and cardia: A review of the disease and its treatment. Ann Surg Oncol 2006; 13: 12-30.

4. Lagergren J, Bergstrom R, Lindgren A, Nyren O. Symptomatic gastroesophageal reflux as a risk factor for esophageal adenocarcinoma. N Engl J Med 1999; 340: 825-31.

5. Macdonald J, Smalley S, Benedetti J, Hundahi S, Estes N, Stemmermann G, et al. Chemoradiotherapy after surgery compared with surgery alone for adenocarcinoma of the stomach or gastroesophageal junction. N Engl J Med 2001; 345: 725-30.

6. Cunningham D, Allum W, Stenning S, Thompson J, Van De Velde C, Nicolson M, et al. Perioperative chemotherapy versus surgery alone for resectable gastroesophageal cancer cancer. N Engl J Med 2006; 355: 11-22.

7. Manterola C, Torres R, Burgos L, Vial M, Pineda V. [Methodological quality of an article on the treatment of gastric cancer adopted as protocol by some Chilean hospitals]. Rev Med Chile 2006; 134: 920-6.

8. Barbour A, Rizk N, Gonen M, Tang L, Bains M, Rusch V, et al. Adenocarcinoma of the gastroesophageal junction: influence of esophageal resection margin and operative approach on outcome. Ann Surg 2007; 246: 1-8.

9. Demeester S. Colon interposition following esophagectomy. Dis Esophagus 2001; 14: 169-72.

10. American Cancer Society. American Joint Committee on Cancer. AJCC Cancer Staging Manual. $6^{\text {th }}$ Edition. Lippincott-Raven. 2002.

11. Kirby J. Quality of life after oesophagectomy: the patient's perspective. Dis Esophagus 1999; 12: 168-71.

12. Lerut T, Nafteux P, Moons J, Coosemans W, Decker G, De Leyn $\mathrm{P}$, et al. Three-field lymphadenectomy for carcinoma of the esophagus and gastroesophageal junction 
in 174 R0 resections: impact on staging, disease-free survival, and outcome: a plea for adaptation of TNM classification in upper-half esophageal carcinoma. Ann Surg 2004; 240: 962-72.

13. Patti M, Corvera C, Glasgow R, Way L. A hospital's annual rate of esophagectomy influences the operative mortality rate. J Gastrointest Surg 1998; 2: 186-92.

14. Gee D, Rattner D. Management of gastroesophageal Tumors. Oncologist 2007; 12: 175-85.

15. Sihvo E, Luostarinen M, Salo J. Fate of patients with adenocarcinoma of the esophagus and the esophagogastric junction: A population-based analysis. Am J Gastroenterol 2004, 99: 419-24.

16. Von Rahden B, Stein H, Siewert J. Surgical management of esophagogastric junction tumors. World J Gastroenterol 2006; 12: 6608-13.

17. Waters J, Norman A, Cunningham D, Scarffe J, Webb A, Harper P, et al. Long-term survival after epirubicin, cisplatin and fluorouracil for gastric cancer: results of a randomized trial. Br J Cancer 1999; 80: 269-72.

18. http://www.redsalud.gov.cl/archivos/guiasges/Guia_ GES_sobre_cancer_gastrico20062.pdf. 